\title{
Finite difference scheme for a high order nonlinear Schrödinger equation with localized damping
}

\author{
Marcelo M. Cavalcanti, Wellington J. Corrêa, Mauricio A. Sepúlveda \\ C. and Rodrigo Véjar Asem
}

\begin{abstract}
In this work we present a finite difference scheme used to solve a High order Nonlinear Schrödinger Equation. These equations can model the propagation of solitons travelling in fiber optics ([3], [11]). The scheme is designed to preserve the numerical energy at $L^{2}$ level, and control the energy at $H^{1}$ level for a suitable choose on the equation's parameters. We show numerical results displaying conservation properties of the schemes using solitons as initial conditions.
\end{abstract}

Mathematics Subject Classification (2010): 35Q55, 65-06, 65M06, $65 \mathrm{Z} 05$.

Keywords: High order, nonlinear Schrödinger equation, localized damping, dissipation, finite difference methods.

\section{Introduction}

We will study a numerical solution of a Higher order Non-Linear Schrödinger (HNLS) equation over an interval $\Omega:=[0, L]$ :

$$
\left\{\begin{array}{l}
i u_{t}+a_{1} u_{x x}+i a_{2} u_{x x x}+a_{3}|u|^{2} u+i a_{4}|u|^{2} u_{x}+i a_{5} u|u|_{x}^{2}+i a(x) u=0, \Omega \times(0, T) \\
u(0, t)=u(L, t)=0, \quad u_{x}(L, t)=0, t \geq 0 \\
u(x, 0)=u_{0} \text { in } \Omega
\end{array}\right.
$$

This paper has been presented at the fourth edition of the International Conference on Numerical Analysis and Approximation Theory (NAAT 2018), Cluj-Napoca, Romania, September 6-9, 2018. Research of Marcelo M. Cavalcanti partially supported by the CNPq Grant 300631/2003-0. Research of Wellington J. Corrêa partially supported by the CNPq Grant 438807/2018-9. Research of Mauricio Sepúlveda C. was supported FONDECYT grant no. 1180868, and by CONICYT-Chile through the project AFB170001 of the PIA Program: Concurso Apoyo a Centros Científicos y Tecnológicos de Excelencia con Financiamiento Basal.

Rodrigo Véjar Asem acknowledges support by CONICYT-PCHA/Doctorado Nacional/201521150799. 
where $a_{1}, a_{2}, a_{3}, a_{4}, a_{5} \in \mathbb{R}, a_{1}, a_{3} \geq 0$, and $u=u(x, t)$ is a complex valued function. The damping function $a(x)$ belongs to $L^{\infty}(\Omega)$ and assumed to be such that

$$
a(x) \geqslant a_{0}>0 \text { a.e. in an open, non-empty subset } \omega \text { of }(0, L),
$$

where it is acting effectively. This equation plays an important rule in soliton theory. It has applications in the propagation of femtosecond optical pulses in a monomode optical fiber, accounting for additional effects such as third order dispersion, self-steeping of the pulse, and self-frequency shift [11]. When $a(x)=0$, we can also consider equation (1.1) as a generalization of the classical Nonlinear Schrödinger (NLS) equation

$$
i u_{t}+a_{1} u_{x x}+a_{2}|u|^{2} u=0
$$

which can be obtained using $a_{3}=a_{4}=a_{5}=0$ in (1.1). This equation describes the electric field envelope of a laser beam in a medium with Kerr nonlinearity [7]. It is also known in plasma physics, where it describes Langmuir waves in a plasma with non-homogeneous density [10]. If in (1.1) we also take $a_{1}=a_{2}=0, a_{3}=1$, $a_{5}=0$ and $a_{4}=6$, we can obtain a modified Korteweg-de Vries (KdV) equation which studies, for example, surface waves on conducting nonviscous incompressible liquid under the presence of a transverse electric field [16]. The KdV equation has also great importance in the study of surface water waves [12]. In this sense, numerically solving (1.1) can also solve many subproblems derived from it.

When considering $a(x)=0$, Carvajal proved in [4] for $a_{3} a_{5} \neq 0$ the global well-posedness of the Cauchy Problem (1.1) in $H^{s}(\mathbb{R}), s>\frac{1}{4}$ when $3 a_{2} a_{3}=a_{1} a_{4}$. Meanwhile, Takaoka proved in [22], for $a_{3}=1$, the local well-posedness for the Cauchy Problem (1.1) in $H^{s}(\mathbb{T}), s>\frac{1}{2}$, where $\mathbb{T}$ is a unidimensional torus. Similar conclusions were obtained also by Takaoka in [21] for $a_{3}=0$, where the well-posedness is over $H^{\frac{1}{2}}(\mathbb{R})$. Regularity properties were studied by Alves et al. [2] when $a_{4}=a_{5}=0$.

Exact solutions for (1.1) can be found using the Inverse Scattering Transform (IST) [1], proposed originally in Zakharov et al. [23]. Its integration depends on the values of $a_{3}, a_{4}$ and $a_{5}$. In particular: for $a_{1}=\frac{1}{2}, a_{2}=1$, and rewriting equation (1.1) as

$$
i u_{t}+\frac{1}{2} u_{x x}+|u|^{2} u+i \varepsilon\left(\beta_{1} u_{x x x}+\beta_{2}|u|^{2} u_{x}+\beta_{3}|u|_{x}^{2} u\right)=0
$$

where $\beta_{1}, \beta_{2}, \beta_{3}, \varepsilon$ are real constants, then exact solutions can be obtained via IST for the following cases:

- For the derivative NLS equation of type I: $\beta_{1}: \beta_{2}: \beta_{3}=0: 1: 1$ [3].

- For the derivative NLS equation of type II: $\beta_{1}: \beta_{2}: \beta_{3}=0: 1: 0$ [5].

- For the Hirota equation: $\beta_{1}: \beta_{2}: \beta_{3}=1: 6: 0$ [9].

- For the Sasa-Satsuma equation: $\beta_{1}: \beta_{2}: \beta_{3}=1: 6: 3[18]$.

Exact solutions are all of solitonic form. $N$-soliton solutions can also be obtained [9]. Potasek [17] shows some particular solutions that has been proven experimentally. But even when continuous solutions can be found for some specific initial conditions and some values for the real constans in (1.1), numerical solutions can prescinde from those requirements when computed. We can even use non-solitonic initial conditions in order to obtain a result. One way to compute numerical solutions is using the Finite 
Difference Method, whose computational implementation can be done in an fast and efficient way.

Other ways to obtain numerical solutions for (1.1) has been studied by different authors in the recents years. One of the first scheme were proposed by Delfour, Fortin and Payre [6], which solves the NLS equation (1.3) proposing a rule to discretize powers of the nonlinearity multiplying the $a_{2}$ term. Their method has a strong property: it preserves the discrete versions of both the $L^{2}$ norm and the energy of the numerical solution, where their continuous versions are given by the following relations:

$$
\begin{aligned}
& \|u\|_{L^{2}(\Omega)}^{2}(t)=\int_{\Omega}|u(x, t)|^{2} d x \\
& E(t):=\frac{\alpha}{2} \int_{\Omega}|\nabla u(x, t)|^{2} d x-\frac{\gamma}{4} \int_{\Omega}|u(x, t)|^{4} d x
\end{aligned}
$$

for $u=u(x, t) \in \Omega \subset \mathbb{R} \times \mathbb{R}^{+} \longmapsto \mathbb{C}$ the exact solution of (1.1). The convergence of the numerical method is proved in Matsuo and Furihata [8]. Pazoto et al [15] proposed a finite difference scheme which solves the critical generalilzed Kortewetgde Vries equation $(\mathrm{GKdV}-4)$ in a bounded domain. The higher-power term $u^{4} u_{x}$ was rewritten as a linear combination of other derivatives in order to obtain specific conservation properties. Smadi and Bahloul [19] [20] combined a Compact Padé Finite Difference scheme [13] with a fourth order Runge-Kutta (RK4) scheme. They splitted the problem in two parts: a linear one which is solved using the finite difference scheme; and a nonlinear, which is solved using the RK4 scheme. The method was implemented with an interesting success, but no analysis of the error, convergence, or preserved quantities was made. We will compare their proposal with our method later on.

The structure of this work is as follows: Section 2 shows the numerical scheme we propose, along with some notation and its properties; Section 3 will present results for some experiments; and Section 4 contains conclusions regarding the results obtained.

\section{Numerical scheme}

\subsection{Notation}

Because of the boundary conditions given in Problem (1.1), and for the sake of the following analysis, we will introduce the vector space for a $M \in \mathbb{N}$ given:

$$
X_{M}:=\left\{u=\left[\begin{array}{llll}
u_{0} & u_{1} & \ldots & u_{M}
\end{array}\right]^{T} \in \mathbb{C}^{M+1}: u_{0}=u_{M-1}=u_{M}=0\right\}
$$

Let us introduce the classical finite differences operators for complex-valued arrays:

$$
\begin{aligned}
& {\left[\boldsymbol{D}^{+} u\right]_{j}:=\frac{u_{j+1}-u_{j}}{\Delta x},\left[\boldsymbol{D}^{-} u\right]_{j}:=\frac{u_{j}-u_{j-1}}{\Delta x}} \\
& \boldsymbol{D} u:=\frac{1}{2}\left(\boldsymbol{D}^{+} u+\boldsymbol{D}^{-} u\right) \\
& \boldsymbol{D}^{2} u:=\boldsymbol{D}^{+} \boldsymbol{D}^{-} u, \boldsymbol{D}^{3} u:=\boldsymbol{D} \boldsymbol{D}^{+} \boldsymbol{D}^{-} u
\end{aligned}
$$


For $u, v \in X_{M}$, and $\Delta x:=\frac{L}{M+1}$, let us consider the discrete space $L^{2}(0, L)_{\Delta}$ of complex-valued vectors endowed with the inner product

$$
(u, v)_{2}:=\sum_{j=0}^{M} u_{j} \bar{v}_{j} \Delta x
$$

this induces a discrete version of the $L^{2}$ norm:

$$
\|u\|_{2}^{2}:=(u, u)_{2} \text {. }
$$

and hence, at a timestep $n$, we define a numerical energy at $L^{2}$ level as follows:

$$
E_{L^{2}}\left(u^{n}\right):=\frac{1}{2}\left\|u^{n}\right\|_{2}^{2}
$$

Here we will write the functions that will approximate Problem (1.1). The linear terms will be approximated using the classical finite differences operators given in (2.1). The nonlinear terms are approximated as follows: for the term multiplied by $a_{3}$, we will use the method proposed in Delfour et al. [6]; this is:

$$
\left|u\left(x_{j}, t_{n}\right)\right|^{2} u\left(x_{j}, t_{n}\right) \approx\left|u_{j}^{n+\frac{1}{2}}\right|^{2}\left(u_{j}^{n+\frac{1}{2}}\right)
$$

For the term multiplied by $a_{4}$, we define

$$
\begin{aligned}
& F_{a_{4}}: \mathbb{C}^{M} \longrightarrow \mathbb{C}^{M} \\
& u_{j}^{(p)} \longrightarrow\left[F_{a_{4}}\left(u^{(p)}\right)\right]_{j}:=\frac{1}{2}\left|\frac{u_{j}^{p}+u_{j}^{n}}{2}\right|^{2} D\left(\frac{u_{j}^{p}+u_{j}^{n}}{2}\right) \\
& +\frac{1}{2} \boldsymbol{D}\left(\left|\frac{u_{j}^{p}+u_{j}^{n}}{2}\right|^{2} \frac{u_{j}^{p}+u_{j}^{n}}{2}\right) \\
& -\frac{1}{2} \frac{u_{j}^{p}+u_{j}^{n}}{2} D\left(\left|\frac{u_{j}^{p}+u_{j}^{n}}{2}\right|^{2}\right)
\end{aligned}
$$

The $a_{5}$ term will be discretized directly; this is, we define

$$
\begin{aligned}
& F_{a_{5}}: \mathbb{C}^{M} \longrightarrow \mathbb{C}^{M} \\
& u_{j}^{(p)} \longrightarrow\left[F_{a_{5}}\left(u^{(p)}\right)\right]_{j}:=\left(\frac{u_{j}^{p}+u_{j}^{n}}{2}\right) D\left(\left|\frac{u_{j}^{p}+u_{j}^{n}}{2}\right|^{2}\right)
\end{aligned}
$$

The given functions were defined in such a way that the numerical energy at $L^{2}$ level is conserved when $a(x)=0, x \in \Omega$. Our proposal then reads as follows: $\forall j \in\{1, \ldots, M\}$, $\forall n \in \mathbb{N}$, and for a given $u^{0} \in X_{M}$ the numerical scheme will be given component-wise by

$$
\left\{\begin{array}{l}
i D_{t} u_{j}^{n}+a_{1} D^{2}\left(u_{j}^{n+\frac{1}{2}}\right)+i a_{2} D^{3}\left(u_{j}^{n+\frac{1}{2}}\right)+a_{3}\left|u_{j}^{n+\frac{1}{2}}\right|{ }^{2} u_{j}^{n+\frac{1}{2}} \\
\quad+i a_{4}\left[F_{a_{4}}\left(u^{(n+1)}\right)\right]_{j}+i a_{5}\left[F_{a_{5}}\left(u^{(n+1)}\right)\right]_{j}+a\left(x_{j}\right) u_{j}^{n+\frac{1}{2}}=0 \\
u^{n} \in X_{M}, \quad \forall n \in \mathbb{N} \\
u^{0} \in X_{M} \text { given. }
\end{array}\right.
$$


where $u_{j}^{n} \approx u\left(x_{j}, t_{n}\right)$ is the approximation of the exact solution $u(x, t)$ at the time $t_{n}=n \Delta t$ and at the coordinate $x_{j}=j \Delta x$; and $u_{j}^{n+\frac{1}{2}}:=\frac{1}{2}\left(u_{j}^{n+1}+u_{j}^{n}\right)$. At each timestep, the scheme leads us to solve a linear system of equations, where the matrix involved is pentadiagonal; and a nonlinear problem solved using a Picard fixed-point iteration, a procedure similar to the one proposed in Delfour, Fortin, and Payre [6], which in turn induces us to use a small $\Delta t$ in order to guarantee the contraction of the operator involved. The numerical scheme (2.4) has the following main properties:

Theorem 2.1. Let $u^{0} \in X_{M}:\left\|u^{0}\right\|_{2}^{2}<\infty$. If $a(x)=0, \forall x \in \Omega$, then, $\forall n \in \mathbb{N}$, and for $u^{n} \in X_{M}$, we have

$$
E_{L^{2}}\left(u^{n+1}\right)=E_{L^{2}}\left(u^{n}\right)
$$

On the other hand, we will consider the energy at $H^{1}$-level of the numerical solution at a timestep $n$ as follows

$$
E_{H^{1}}\left(u^{n}\right):=\frac{a_{1}}{2}\left\|D^{+} u^{n}\right\|_{2}^{2}-\frac{a_{3}}{4}\left\|u^{n}\right\|_{4}^{4}
$$

where

$$
\Delta E_{H^{1}}:=\max _{n, m \in \mathbb{N}}\left|E_{H^{1}}\left(u^{n}\right)-E_{H^{1}}\left(u^{m}\right)\right|
$$

Then, we have:

Theorem 2.2. Let $u^{n} \in X_{M}$ the numerical solution of (1.1) using scheme (2.4) using $a(x)=0, \forall x \in \Omega$. If in (1.1) $3 a_{2} a_{3}=a_{1}\left(a_{4}+2 a_{5}\right)$, and if $C=\frac{1}{2}$ in (2.3), then the following property holds

$$
E_{H^{1}}\left(u^{n+1}\right)=E_{H^{1}}\left(u^{n}\right)+\mathcal{O}\left(\Delta t\left(\Delta t^{2}+\Delta x^{2}\right)\right)
$$

When a damping function $a(x)$ is present, then we have the following property:

Theorem 2.3. Consider a sequence $\left\{u^{n}\right\}_{n \in \mathbb{N}} \subset X_{M}$ induced by the numerical scheme (2.4), and consider the function $a(x)$ and the set $\omega$ as defined in (1.2). If $u^{0} \in$ $L^{2}(0, L)_{\Delta}, 6 a_{3} \geq\left|3 a_{4}+2 a_{5}\right|$ or $3 a_{4}+2 a_{5}=0$, and for $T_{0}=n \Delta t>0$, there exist a positive constant $C=C\left(T_{0}\right)$ and $\mu=\mu\left(T_{0}\right)$, both independent of $\Delta x$ and $\Delta t$, such that the inequality

$$
E_{L^{2}}^{n} \leq C\left\|u^{0}\right\|_{2}^{2} e^{-\mu n \Delta t}
$$

holds for all $n>0$.

\section{Numerical results}

In this section, we will start by comparing our scheme with previous works. We will then show some numerical experiments performed using a FORTRAN code which implements scheme (2.4). Given the particular interest on physical applications, our simulations will be performed over a domain $\Omega=[a, b], a, b \in \mathbb{R}$ instead of the interval $[0, L]$. The results proved on this paper will still hold if we use a suitable coordinates change of the kind of $x=\tilde{x}-a, \tilde{x} \in[a, b]$, where the variable change is such that $L=b-a$. 


\subsection{Comparison with previous works}

The only known work dealing with numerical methods for Problem (1.1) was proposed by Smadi and Bahloul [19] (from now on: SB scheme), which was implemented again in [20] with other examples. Their scheme splits Problem (1.1) in two parts: a linear one, which is solved using a Compact Finite Difference scheme [13]; and a nonlinear part, which solves the problem through an explicit fourth-order Runge-Kutta method.
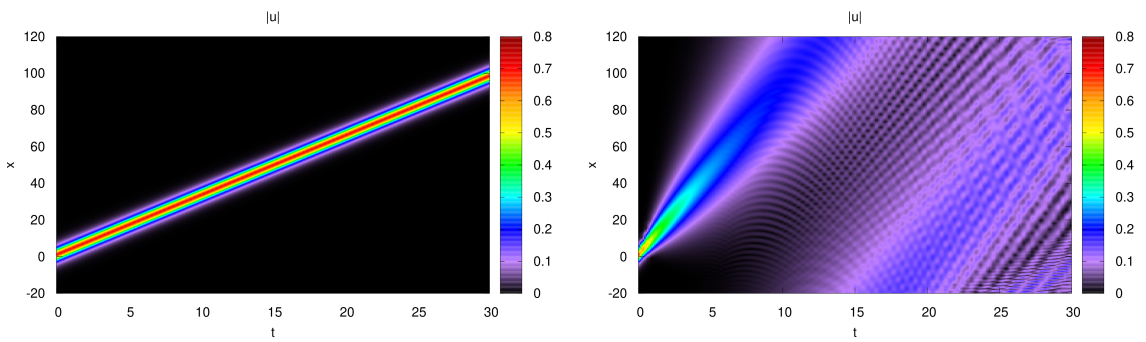

Figure 1. Numerical approximation when using (3.1) with $t=0$ as initial condition, obtained by: at left, numerical scheme (2.4); at right, SB scheme.

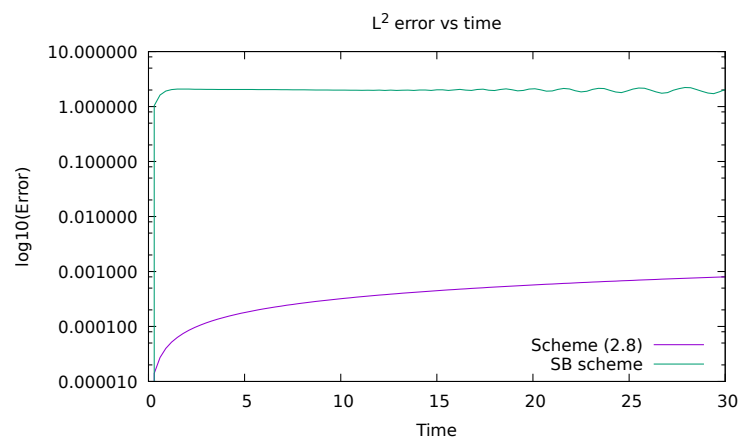

FiguRE 2. Semi-log plot of the comparison of the numerical error produced by both schemes.

In this subsection, we will compare the results computed by our proposal and the SB scheme for a particular case. To this end, we will solve equation (1.1) without damping term; this is, using $a(x)=0, \forall x \in \Omega$. We will use the following solution obtained by $\mathrm{Li}$ [14]:

$$
u(x, t)=\frac{i \rho}{\cosh _{q}(\eta(x-\chi t))} e^{i(k t-\tilde{\Omega} x)}, \quad x \in \Omega:=[-40,150], \quad t \in[0,30]
$$

where, in (1.1), $a_{1}=3, a_{2}=3, a_{3}=1, a_{4}=3$; while in (3.1), $\alpha_{3}=-a 3, \alpha_{4}=-a 4$, 


$$
\begin{aligned}
a_{5}=-\left(\alpha_{5}+\alpha_{4}\right) & =0, \eta=\frac{1}{2}, q=3, \tilde{\Omega}=a_{2} / \alpha_{4}, k=-2 \tilde{\Omega}^{3} \alpha_{3}, \chi=\alpha_{3}\left(\eta^{2}+3 \tilde{\Omega}^{2}\right), \\
\rho & =\sqrt{\frac{6 \alpha_{3} \eta^{2} q}{\alpha_{4}}} \text { and } \cosh _{q}(\xi):=\frac{e^{\xi}-q e^{-\xi}}{2} .
\end{aligned}
$$

Figure 1 shows the results using the numerical scheme given in (2.4) using $\Delta t=$ $10^{-3}$ and $\Delta x=\frac{190}{2^{14}} \approx 0.0116$. Because there is no damping function, we expect the numerical preservation of the energy at levels $L^{2}$ and $H^{1}$. For our numerical scheme, we have that $\Delta E_{H^{1}}=7.176423 \cdot 10^{-8}$, while $\Delta E_{L^{2}}=6.883855 \cdot 10^{-10}$. The computation time was $450.835999 \mathrm{~s}$. Meanwhile, Figure 2 shows what is obtained using the SB scheme for the same mesh and timestep used for the results in Figure 1. Here, we have that $\Delta E_{H^{1}}=175.938612, \Delta E_{L^{2}}=9.723801 \cdot 10^{-4}$. The computation time was $271.823999 \mathrm{~s}$.

Remark 3.1. The paper of Smadi and Bahloul [19] does not give details regarding the discretization of the first-order derivatives in non-linear terms (the description of the Runge-Kutta method is presented in [19] in terms of a $f(u)$, and not of a $f\left(u, u_{x}\right)$ ). We assume in our simulations a finite deference centered as an approximation of $u_{x}$, however, it is worth noting that we tested with other more efficient approaches obtaining very similar results for the SB method that we show here.

\subsection{Some numerical experiments}

In this subsection of numerical examples we show some original results that test the adaptability of our numerical scheme for different situations.

3.2.1. First example: Effects of a strong damping. We assume the following initial condition $u(x, 0)=u_{0} \operatorname{sech}(k x)$, where $k=1$ and $u_{0}=\sqrt{6}$. We consider additionally, that, $a_{1}=3, a_{2}=1, a_{3}=0.03, a_{4}=0.1, a_{5}=-0.05$, and $a(x) \equiv 0$ (that is, without damping term). Then an exact solution of (1.1) is obtained, which corresponds to a soliton of a hyperbolic secant squared pulses often referred to as "bright" pulses (see for more details Potasek and Tabor [17]). Now, the effect that we want to show in this example is what happens with this solution when adding a strong damping term. For that, we introduce a damping function concentrated in a neighborhood of the boundary of the spatial interval, given by

$$
a(x)= \begin{cases}1000, & x \in(-15,-10) \cup(10,15) \\ 0, & \text { in other case }\end{cases}
$$

In our computations, $t \in[0,1000], x \in[-15,15], \Delta t=0.00001$ and $\Delta x=\frac{30}{2^{13}} \approx$ 0.00366. The form of the travelling soliton can be found in Figure 4. First, we observe that in the first times the wave propagates as the hyperbolic secant soliton predicted in Potasek and Tabor [17], while does not touch the support of the damping function. However, once the soliton approaches the area of influence (approximately at $t=180$ ), the damping function is so high that the soliton gets reflected instead of proceeding with his original path. In each reflection the soliton loses energy at $L^{2}$ level following the exponential rate predicted in the previous theorems, and illustrated in Figure 3 left. The energy at $H^{1}$ level also decays at an exponential rate in each reflection. 

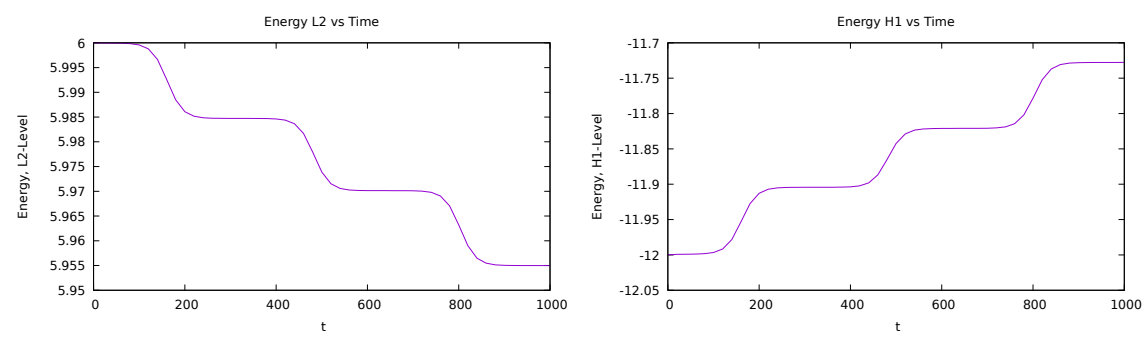

Figure 3. First case results. Left: time evolution of the $L^{2}$ energy. Right: time evolution of the $H^{1}$ energy.

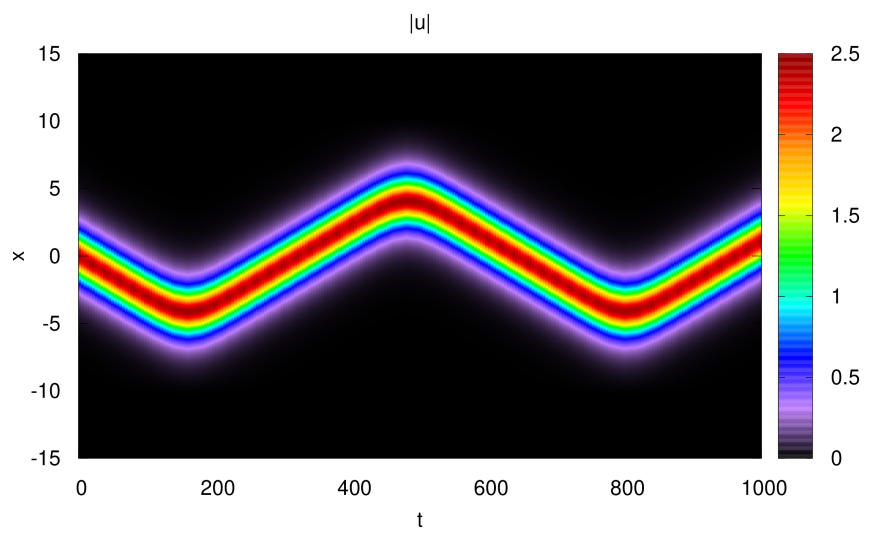

Figure 4. Time evolution of the travelling soliton for the first case.

3.2.2. Second example: Crossing of solitons. In this second example, we will simulate a cross between two solitons. For this we consider the exact solution of Hirota described in [9]. Then we consider our numerical scheme to approximate the Hirota equation:

$$
i u_{t}+u_{x x}+|u|^{2} u+i \frac{1}{10} u_{x x x}+i \frac{3}{10}|u|^{2} u_{x}=0 .
$$

for $(x, t) \in[-50,50] \times(0,15]$. At that time, we consider as initial condition, the solution of Hirota [9] for $t=0$, that approximately corresponds to the sum of two hyperbolic secants of different amplitudes and centered in distant points. In this way, we calculate the numerical solution described in section 2 of this paper and we compare the result with the exact solution described in Hirota [9].

Given the absence of a damping function and because $3 a_{2} a_{3}=a_{1} a_{4}$, we conclude that there should not exist energy decay at $L^{2}$ and $H^{1}$ levels. For our calculations, we have made $\Delta t=0.0001$, and $\Delta x=\frac{100}{2^{15}} \approx 0.00305$. 

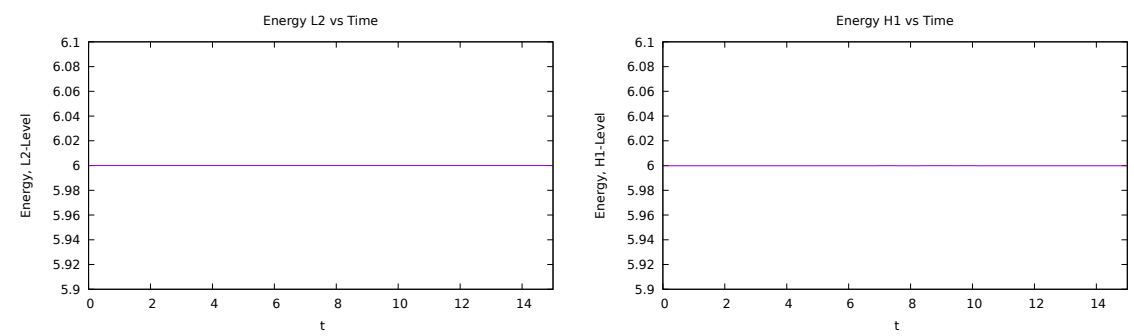

Figure 5. Preserved quantities for the 2 soliton experiment (second case). Left: $L^{2}$ level energy. Right: $H^{1}$ level energy.
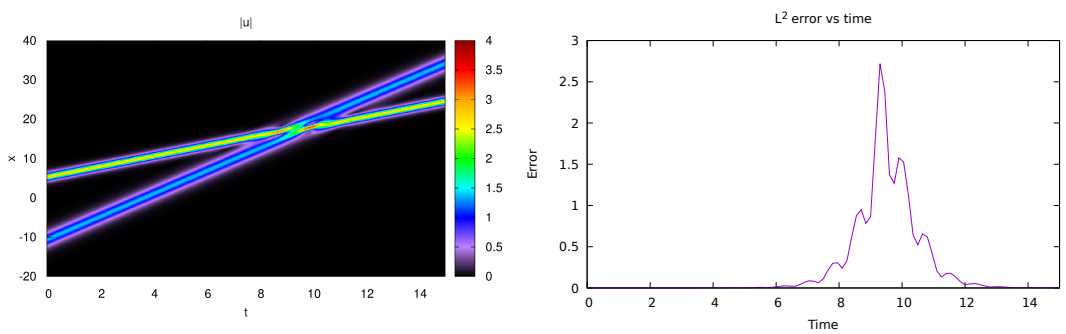

FiguRE 6. Left: the 2 soliton solution over time. Right: numerical error.

Regarding the error, we observed that the shape of the numerical solution moves away from the exact solution just at the moment of crossing between both solitons (see Figure 6 right). However, surprisingly we can notice that past the crossing, the numerical solution returns to reasonable levels of error $(t>13)$. The time evolution of the preserved quantities can be seen in Figure 5, where $\Delta E_{L^{2}}=1.216 \times 10^{-8}$ and $\Delta E_{H^{1}}=4.088 \times 10^{-5}$.

\subsection{Computational performance}

The computational performance will be now discussed. Using the same solution (3.1) from the previous subsection, Tables 1 and 2 illustrate how our scheme performs for two different timesteps; $\Delta t=10^{-3}$ and $\Delta t=10^{-4} . \Delta t=10^{-2}$ was not included because it fails to guarantee the contraction condition of the operator involved in the nonlinear problem (2.4). For our computations, we have used a home computer equipped with a Linux operative system, an Intel Core i5-2400 chip with 4 processors at $3.10 \mathrm{GHz}$ each, and $9.7 \mathrm{~GB}$ of RAM memory. Parallelization was not implemented. From both tables, we see that the decrease of the numerical error is evident. We can also see a real improvement on the preservation of the $H^{1}$ energy. 


\begin{tabular}{ccccc}
$\Delta x$ & $\|e\|_{L^{2}\left(0, T ; L^{2}(\Omega)\right)}^{2}$ & $t_{\text {comp }}[\mathrm{s}]$ & $\Delta E_{L^{2}}$ & $\Delta E_{H^{1}}$ \\
\hline $190 / 2^{10} \approx 1.885 \mathrm{E}-1$ & $4.948753 \mathrm{E}-1$ & 25.951 & $3.519406 \mathrm{E}-12$ & $6.137745 \mathrm{E}-3$ \\
$190 / 2^{11} \approx 9.277 \mathrm{E}-2$ & $3.024600 \mathrm{E}-2$ & 52.292 & $4.636734 \mathrm{E}-12$ & $3.440563 \mathrm{E}-4$ \\
$190 / 2^{12} \approx 4.638 \mathrm{E}-2$ & $1.874793 \mathrm{E}-3$ & 118.843 & $2.009226 \mathrm{E}-11$ & $2.076023 \mathrm{E}-5$ \\
$190 / 2^{13} \approx 2.319 \mathrm{E}-2$ & $1.163071 \mathrm{E}-4$ & 230.372 & $6.786449 \mathrm{E}-11$ & $1.250147 \mathrm{E}-6$ \\
$190 / 2^{14} \approx 1.159 \mathrm{E}-2$ & $7.168988 \mathrm{E}-6$ & 450.836 & $6.883855 \mathrm{E}-10$ & $7.176423 \mathrm{E}-8$ \\
$190 / 2^{15} \approx 5.798 \mathrm{E}-3$ & $4.869383 \mathrm{E}-7$ & 1162.440 & $1.334082 \mathrm{E}-9$ & $4.808387 \mathrm{E}-9$ \\
\hline
\end{tabular}

TABLE 1. Computational performance using (3.1) as initial condition and reference solution for $\Delta t=0.001$.

\begin{tabular}{ccccc}
$\Delta x$ & $\|e\|_{L^{2}\left(0, T ; L^{2}(\Omega)\right)}^{2}$ & $t_{\text {comp }}[\mathrm{s}]$ & $\Delta E_{L^{2}}$ & $\Delta E_{H^{1}}$ \\
\hline $190 / 2^{10} \approx 1.885 \mathrm{E}-1$ & $4.949154 \mathrm{E}-1$ & 221.080 & $2.506295 \mathrm{E}-11$ & $6.141681 \mathrm{E}-3$ \\
$190 / 2^{11} \approx 9.277 \mathrm{E}-2$ & $3.025991 \mathrm{E}-2$ & 442.384 & $2.235878 \mathrm{E}-12$ & $3.449260 \mathrm{E}-4$ \\
$190 / 2^{12} \approx 4.638 \mathrm{E}-2$ & $1.878424 \mathrm{E}-3$ & 1099.167 & $9.875344 \mathrm{E}-11$ & $2.097018 \mathrm{E}-5$ \\
$190 / 2^{13} \approx 2.319 \mathrm{E}-2$ & $1.171612 \mathrm{E}-4$ & 2168.396 & $2.507041 \mathrm{E}-6$ & $2.301061 \mathrm{E}-6$ \\
$190 / 2^{14} \approx 1.159 \mathrm{E}-2$ & $7.316259 \mathrm{E}-6$ & 3558.420 & $5.695187 \mathrm{E}-10$ & $8.371821 \mathrm{E}-8$ \\
$190 / 2^{15} \approx 5.798 \mathrm{E}-3$ & $4.567125 \mathrm{E}-7$ & 7768.200 & $1.664837 \mathrm{E}-9$ & $2.460758 \mathrm{E}-9$ \\
\hline
\end{tabular}

TABLE 2. Computational performance using (3.1) as initial condition and reference solution for $\Delta t=0.0001$.

\section{Conclusion}

In this work, we have proposed a new way to solve equation (1.1) using a finite difference method. The procedure involved the re-writing of a particular nonlinearity as a convex combination in order to get the conservation of the numerical energy at $L^{2}$ level when no damping term is present. The energy at $H^{1}$ level can also be controlled for sufficiently small values of $\Delta t$ and $\Delta x$. When the damping term is present, the $L^{2}$ energy decays exponentially with time. We have also compared our proposal with the one from Smadi and Bahloul, observing an evident difference between both outputs. We deduce that our numerical method adapts better and more efficiently to the numerical resolution of the HNSL equation with respect to the known methods in the literature (see Smadi and Bahloul [19]), for various examples, with or without damping. Our method can also compute reasonable results using a small computer time at a home PC. Nevertheless, there is still room for improvement; in particular, about the contrast between the numerical and the exact solution when a collision between solitons happens. Also, the Picard iteration can be modified in order to be able to perform more calculations for smaller timesteps. Further studies are needed in both regards. 


\section{References}

[1] Ablowitz, M.J., Segur, H., Solitons and the Inverse Scattering Transform, SIAM, 1981.

[2] Alves, M., Sepúlveda, M., Vera, O., Smoothing properties for the higher-order nonlinear Schrödinger equation with constant coefficients, Nonlinear Anal., 71(2009), 948-966.

[3] Anderson, D., Lisak, M., Nonlinear asymmetric self-phase modulation and selfsteepening of pulses in long optical waveguides, Phys. Rev. A, 27(1983), no. 3, 1393-1398.

[4] Carvajal, X., Sharp global well-posedness for a higher order Schrödinger equation, J. Fourier Anal. Appl., 12(2006), no. 1, 53-70.

[5] Chen, H.H., Lee, Y.C., Liu, C.S., Integrability of nonlinear Hamiltonian systems by inverse scattering method, Phys. Scr., 20(1979), 490-492.

[6] Delfour, M., Fortin, M., Payre, G., Finite-difference solutions of a non-linear Schrödinger equation, J. Comput. Phys., 44(1981), 277-288.

[7] Fibich, G., Adiabatic law for self-focusing of optical beams, Opt. Lett., 21(1996), no. 21, 1735-1737.

[8] Furihata, D., Matsuo, T., Discrete Variational Derivative Method: A StructurePreserving Numerical Method for Partial Differential Equations, Chapman \& Hall/CRC, 2011.

[9] Hirota, R., Exact envelope-soliton solutions of a nonlinear wave equation, J. Math. Phys., 14(1973), no. 7, 805-809.

[10] Kivshar, Y., Malomed, B., Dynamics of solitons in nearly integrable systems, Rev. Modern Phys., 61(1989), no. 4, 763-915.

[11] Kodama, Y., Hasegawa, A., Nonlinear pulse propagation in a monomode dielectric guide, IEEE J. Quantum Electron., QE-23(1989), 510-524.

[12] Korteweg, D.J., De Vries, G., On the change of form of long waves advancing in a rectangular canal, and on a new type of long stationary waves, Philos. Mag., 539(1895), 422-443.

[13] Lele, S.K., Compact finite difference schemes with spectral-like resolution, J. Comput. Phys., 103(1992), 16-42.

[14] Li, B., Exact soliton solutions for the higher-order nonlinear Schrödinger equation, Int. J. Modern Phys. C, 16(2005), no. 8, 1225-1237.

[15] Pazoto, A.F., Sepúlveda, M., Vera, O., Uniform stabilization of numerical schemes for the critical generalized Korteweg-de Vries equation with damping, Numer. Math., 116(2010), 317-356.

[16] Perel'man, T.L., Fridman, A.Kh., El'yashevich, M.M., A modified Korteweg-de Vries equation in electrohydrodynamics, Sov. Phys. JETP, 39(1974), no. 4, 643-646.

[17] Potasek, M.J., Tabor, M., Exact solutions for an extended nonlinear Schrödinger equation, Phys. Lett. A, 154(1991), no. 9, 449-452.

[18] Sasa, N., Satsuma, J., New-type of soliton solutions for a higher-order nonlinear Schrödinger equation, J. Phys. Soc. Jpn., 60(1991), no. 2, 409-417.

[19] Smadi, M., Bahloul, D., A compact split step Padé scheme for higher-order nonlinear Schrödinger equation (HNLS) with power law nonlinearity and fourth order dispersion, Comput. Phys. Commun., 182(2011), 366-371.

[20] Smadi, M., Bahloul, D., Dynamic of HNLS solitons using compact split step Padé scheme, J. Phys. Conf. Ser., 574(2015). 
[21] Takaoka, H., Well-posedness for the one-dimensional nonlinear Schrödinger equation with the derivative nonlinearity, Adv. Differential Equations, 4(1999), no. 4, 561-580.

[22] Takaoka, H., Well-posedness for the higher order nonlinear Schrödinger equation, Adv. Math. Sci. Appl., 10(2000), no. 1, 149-171.

[23] Zakharov, V.E., Shabat, A.B., Exact theory of two-dimensional self-focusing and onedimensional self-modulation of waves in nonlinear media, Sov. Phys. JETP, 34(1972), no. $1,62-69$.

Marcelo M. Cavalcanti

Department of Mathematics, State University of Maringá

87020-900, Maringá, PR, Brazil

e-mail: mmcavalcanti@uem.br

Wellington J. Corrêa

Academic Department of Mathematics, Federal Technological University of Paraná

Campuses Campo Mourão, 87301-899, Campo Mourão, PR, Brazil

e-mail: wcorrea@utfpr.edu.br

Mauricio A. Sepúlveda C.

$\mathrm{CI}^{2} \mathrm{MA}$ and Departamento de Ingeniería Matemática

Facultad de Ciencias Físicas y Matemáticas, Universidad de Concepción

Casilla 160-C, Concepción, Chile

e-mail: mauricio@ing-mat.udec.cl

Rodrigo Véjar Asem

$\mathrm{CI}^{2} \mathrm{MA}$ and Departamento de Ingeniería Matemática

Facultad de Ciencias Físicas y Matemáticas, Universidad de Concepción

Casilla 160-C, Concepción, Chile

e-mail: rodrigovejar@ing-mat.udec.cl 